\title{
Factores De Deserción En Los Estudiantes De La Licenciatura En Derecho, Generación 2016-2020, En La Universidad Júarez Autónoma De Tabasco, División Académica Multidisciplinaria De Los Ríos
}

\author{
Reina Isabel Gutiérrez Rodríguez, \\ Carlos Romeo Rodríguez Mazariego, MD \\ Universidad Juárez Autónoma de Tabasco, División Académica \\ Multidisciplinaria de los Ríos. Tenosique, Mexico
}

URL:http://dx.doi.org/10.19044/esj.2020.v16n13p54

\begin{abstract}
Resumen
Las causas de la deserción escolar son multifactoriales, es decir, no se deben a un mismo factor. En éste trabajo se identificó cada uno de los factores de riesgo de deserción, en los alumnos que estudian la licenciatura en derecho a través de la aplicación técnico-documental, recopilando información de los estudiantes que desertan, basándose en los registros de inscripción y sistema de baja, y por otro lado contó con un enfoque cualitativo observando de esta forma los comportamientos y actitudes que tienen éstos. Los resultados demostraron que la deserción se da principalmente por: falta de recursos económicos y orientación vocacional, marginación en el contexto social, problemas en diferentes ámbitos (salud y familia). Profundizando más en la problemática, se puede inclusive hasta abordar el tema de la delincuencia, porque al no haber empleo los individuos buscarán por otro lado para satisfacer sus necesidades, si los jóvenes hoy en día no se preparan, en un futuro transitaran por muchas dificultades monetarias y no solo ellos sino también sus familiares. La deserción escolar es el fenómeno en el cual el estudiante deja de asistir a las aulas y queda fuera del sistema educativo sin recibir título de escolaridad, es un problema pedagógico que afecta al desarrollo de la sociedad.
\end{abstract}

Palabras Clave: Universidad, sociedad, deserción, derecho, impacto 


\title{
Factors of School Dropout in the Students of the Degree in Law, Generation 2016-2020, In the Universidad Júarez Autónoma De Tabasco, División Académica Multidisciplinaria De Los Ríos
}

\author{
Reina Isabel Gutiérrez Rodríguez, \\ Carlos Romeo Rodríguez Mazariego, MD \\ Universidad Juárez Autónoma de Tabasco, División Académica \\ Multidisciplinaria de los Ríos. Tenosique, Mexico
}

\begin{abstract}
The causes of school dropout are multifactorial, that is, they are not due to same factor. This paper focuses on identifying each risk factors of dropout in law students through the application of technical documentation. It involves the process of collecting information from students who drop out based on registration records and school control system. It has a qualitative focus and it aims to ascertain the behaviors and attitudes of deserter students. The results show that students drop out of school mainly due to low economic resources, bad vocational orientation, marginalization in social context, and problems in different areas like health and family. Delving deep into this problem, it can result to crime because in the absence of employment, an individual will search for other ways to meet his needs. If the young people of today don't prepare, they will go through a lot monetary difficulties in the future alongside their relatives. The school dropout is a phenomenon in which students stop attending classes and finally quit the educational system without receiving a school degree. This is basically a pedagogical problem that affects the development of a society.
\end{abstract}

Keywords: University, society, dropout, law, impact

\section{Introduction}

En México desde la década de los 70 la deserción, la reprobación, el bajo rendimiento escolar y la repetición de cursos fueron dificultades que se traducen en un fracaso escolar.

Para los años 80, época en la que México enfrentó crisis y recesión económica, los jóvenes se vieron en la necesidad de dejar las escuelas y 
aplazar sus estudios. Las universidades se masificaron, se afectó el desempeño y bajó la calidad de la docencia.

Aún sin resolver tal situación, en los 90 con la llegada de la globalización, se exigía estar a la par con el resto del mundo, surgieron conceptos de calidad, evaluación y acreditación.

"Las investigaciones acerca de las causas de la deserción son multifactoriales, es por eso que para un mejor estudio se debe de conocer y clarificar cada uno de estos factores." (Liz, 2011)

Por país, México se ubica en la posición 11 de un total de 13 países que proporcionaron información al IIESALC (Instituto Internacional para la Educación Superior en América Latina y el Caribe), sobre el número de adultos que han concluido estudios universitarios hasta septiembre de 2005 en relación con la población total, superando solo a Guatemala y Uruguay. La lista la encabezan Bolivia, Honduras y Venezuela (Martinez, 2006).

Con base en estudios realizados por la OCDE (Organización para la Cooperación y el Desarrollo Económico), de los países miembros de dicha organización, México comparte con Turquía el primer lugar en el abandono de universitarios. Y con base en datos del Instituto Nacional de Estadística y Geografía (INEGI), en México sólo ocho de cada 100 alumnos concluyen una carrera universitaria, la principal razón es la falta de recursos económicos.

Herlinda Suárez, académica del Centro Regional de Investigaciones Multidisciplinarias de la UNAM, indicó que los resultados de la Encuesta Nacional de la Juventud 2010 muestran que 76 por ciento de los jóvenes de 12 a 29 años les gustaría llegar a estudiar una licenciatura, sin embargo, sólo 23 por ciento de los jóvenes entre los 18 y 29 años tienen estudios en ese nivel. De acuerdo con la OCDE, en México sólo el 38 por ciento de los jóvenes que cursan la universidad logran graduarse (Gracia, 2015).

El subsecretario de Educación Superior de la Secretaría de Educación Pública, Fernando Serrano Migallón, afirmó que |durante los últimos 15 años el índice de deserción universitaria se ha ubicado entre $7.5 \%$ y $8.5 \%$ a escala nacional. También explicó que es durante el primer año en que se produce la mayor cantidad de abandonos, por lo que es en el primer año de los y las universitarios en los cuales el gobierno debe intervenir (Serrano, 2013).

Los universitarios están cerca de ser el grupo que más cesa prematuramente sus estudios. En los últimos seis años se registró una tendencia descendente en el índice de eficiencia terminal de los estudiantes de educación superior, al grado que sus cifras están a punto de emparejarse con los de media superior, históricamente el nivel de educación con los índices más bajos. El índice de eficiencia terminal es un indicador que mide el porcentaje de alumnos que concluyen sus estudios en cualquiera de los cuatro niveles educativos: primaria, secundaria, media superior y superior. Durante el c escolar 2012-2013, la porción de estudiantes de educación superior que 
terminó sus estudios fue de 73.7\%. Para el ciclo 2017-2018, cayó a 71.5\%. El índice del nivel medio superior, en contraste, ha ido escalando: subió de $63 \%$ a 66.6\% en el mismo período (Arena, 2018).

En 2012, Corea y México fueron los únicos países que mostraron tasas de desempleo más altas entre los adultos con educación superior $(2.9 \%$ y $4.6 \%$, respectivamente) que entre los adultos sin educación media superior $(2.6 \% \mathrm{y}$ $3.5 \%$, respectivamente). En México, la diferencia es aún más marcada entre los adultos jóvenes (de 25 a 34 años): el 6.7\% de los graduados de educación superior y el $4.5 \%$ de los jóvenes adultos con educación por debajo de la enseñanza media superior están desempleados (Castañeda \& Rebolledo, 2014).

Por otra parte, en el estado de Tabasco a pesar de que los índices de deserción escolar se han logrado disminuir de manera considerable, la falta de recursos económicos, los problemas emocionales, las adicciones, embarazos no deseados y la mala elección de carreras, son los principales factores que ocasionan que jóvenes abandonen sus estudios de nivel medio y superior en la entidad. Lamentablemente sigue siendo un tema que genera preocupaciones y obviamente reacciones, tanto de la administración federal, como estatal. Se han establecido estrategias para reducir la tasa de deserción escolar en ambos niveles, implementado programas muy concretos para reducir el fracaso de las escuelas cuando se va un estudiante. El programa "Yo no abandono", trabaja con los alumnos y los padres de familia para que los jóvenes no deserten de sus planteles educativos. La tasa de deserción en el nivel superior es de 4.75, y anteriormente era de 6.4 por ciento (Ygartua, 2018).

La Universidad Juárez Autónoma de Tabasco (UJAT) registra anualmente el $30 \%$ de deserción, como principales causas se tienen la crisis económica y la falta de vocación de los alumnos, manifiesta la dirección de servicios académicos de la máxima casa de estudios. Lo anterior lo dio a conocer la titular del área, Dora María Frías Márquez, quien aseguró que dicho porcentaje coloca a la universidad dentro de la media nacional. El factor más importante es el económico, los muchachos se ven obligados a veces a abandonar sus estudios para trabajar y apoyar a sus familias, o apoyarse a ellos mismos, estas condiciones se dan a nivel nacional. La secretaria de Servicios Escolares de la UJAT aseveró que la Secretaría de Educación y la propia casa de estudios han instrumentado programas de becas y manutención para estudiantes de tercer semestre en adelante, que es cuando el alumno define si continúa sus estudios (Arias, 2017).

Por desgracia, por la situación económica que se padece a nivel nacional, en Tabasco específicamente en la Universidad Juárez Autónoma de Tabasco aumentó la deserción escolar entre el uno y dos por ciento de la plantilla por no tener recursos para seguir, se busca que, ante un posible 
incremento en el presupuesto, lograr destinar dinero para mayor cantidad de becas y evitar, en lo posible, este problema (Ruiz, 2013).

En las 12 divisiones académicas de la Universidad Juárez Autónoma de Tabasco donde se imparten 53 carreras profesionales, se tiene un alto índice de deserción escolar, por causas multifactoriales, el 50 por ciento de los jóvenes que ingresan a la UJAT con el tiempo se dan de baja sin terminar sus estudios. El nivel de deserción de la Universidad es muy alto si consideramos que en un año se inscribieron 7 mil 400, 7 mil 500, y consideramos el nivel de egresados que fue como de 3 mil 400, estamos hablando de un nivel de deserción de un 50 por ciento que es muy alto. Es multifactorial, generalmente en primer lugar está la falta de recursos económicos que aun cuando la universidad es muy barata en cuanto a sus costos, también es cierto que muchos tienen que viajar (Narváez , 2020).

En la División Académica Multidisciplinaria de los Ríos (DAMR) ubicada en el municipio de Tenosique y la División Académica de Ciencias Sociales y Humanidades (DACSyH), se ofertan la licenciatura en Derecho. Aunque se ha incremento la matrícula en la licenciatura en derecho, el análisis de egreso, muestra que de la cohorte generacional 2010-2015, ingresaron a la Licenciatura 376 estudiantes, de los cuales egresaron 191, quedando un número de 84 estudiantes activos de esa generación, con una deserción de 101 estudiantes. Se detectaron como causales de deserción los problemas económicos, incompatibilidad con la carrera y problemas de salud. También influyen en la retención los cambios de división académica, debido a la distancia que existe entre el municipio de Tenosique y la ciudad capital. Sin duda alguna es importante resaltar que el perfil de ingreso en la mayoría de los casos no se observa ni se cumple al momento del ingreso de los alumnos, debido a ello se tiene deserción escolar, lo cual se aprecia de los estudios que lleva el área de Servicios Escolares, así como de las evaluaciones y entrevistas a través del sistema de tutorías (Rabelo \& Aguilar, 2018).

"Para la cohorte 2010-2015, la DAMR presenta una eficiencia terminal del $51.89 \%$. En la carrera de derecho específicamente fue de $66.67 \%$. y el porcentaje de titulados fue tan solo de 21\%." (Aguilar, 2016)

"Las condiciones económicas de muchos jóvenes son adversas, el acceso a una educación superior es posible gracias a los apoyos otorgados por diversas instituciones, en el año 2017, la licenciatura en derecho de la división multidisciplinaria de los ríos, tuvo la matrícula de 210 alumnos y en ese mismo año solo un $7.5 \%$ de licenciados en derecho obtuvieron su título." (Aguilar, 2017)

"En el año 2018, la licenciatura en derecho tuvo la matrícula de 224 alumnos y a pesar de las becas obtenidas en ese año egresaron el $18 \%$." (Aguilar, 2018) 
"La deserción escolar es un problema social en México, una de las problemáticas vigentes en el sistema educativo, pues, aun cuando se han implementado medidas para combatir el fenómeno, estas son insuficientes, ya que esto se sigue presentando en cada ciclo escolar y dentro de los tres niveles de educación." (Nava, 2018)

"Hay un fuerte problema de rezago y abandono escolar en la trayectoria educativa. De cada 100 niños que ingresan a primaria sólo la mitad termina la instrucción media superior, 21 egresan de una institución universitaria y 13 se titulan." (Vargas, 2013)

La deserción universitaria es un gran conflicto educativo que afecta el desarrollo social y económico de una persona y de todo un país.

A menudo los educadores y administradores de la educación se refieren a la deserción como la enfermedad más aguda del sistema y tratan de diseñar correctivos para hacerla rebajar al mínimo, esto ocurre especialmente en los niveles educativos superiores universitarios. Las razones son contundentes, pues una vez que el estudiante esté en el nivel de educación superior, socialmente se ha realizado un gran esfuerzo económico para tenerlo allí (Paramo \& Correa, 1999).

Ésta es una gran dificultad que aflige claramente a los estudiantes debido a que son los que en un futuro no podrán encontrar un trabajo digno que solvente los gastos propios y familiares, pero no solamente eso, sino que como consecuencia de ello se someterán a vivir en condiciones precarias, inseguras, e inestables, en pocas palabras esta contrariedad traería consigo un futuro infeliz, y desdichado para todo aquel que por diversas cuestiones no pueda concluir sus estudios que como meta se propone en un principio al iniciar la carrera y que no se pueden llevar a cabo por diversos factores como los económicos, familiares, de orientación vocacional y de salud. Lo peor de todo es que esta situación no solo afecta al estudiante sino a todo el país, por que como se sabe un país sin jóvenes preparados profesionalmente seguramente no contará con un buen desarrollo económico, habrá mano de obra sin la cualificación necesaria además habrá un atraso en el desarrollo científico y tecnológico y por ende habrá pobreza extrema en todo el país perjudicando así a las familias y a la sociedad que de él formen parte.

El fracaso escolar es en cualquier caso una catástrofe, absolutamente desoladora en plano moral, humano y social, que muy a menudo genera exclusiones que marcaran a los jóvenes durante toda su vida de adultos. A nivel personal, la deserción y abandono producen desarraigo, soledad, ausencia de ritos, carencia de rutinas y perdida de la capacidad de negociación con otros, soledad social. La deserción no solo involucra a los abandonantes sino también a los abandonados, es un problema que nos atañe a todos (Paramo \& Correa, 1999). 
Se puede entender la deserción como una situación a la que se enfrenta un estudiante cuando aspira y no logra concluir su proyecto educativo, considerándose como desertor a aquel individuo que siendo estudiante de una institución de educación superior no presenta actividad académica durante dos semestres académicos consecutivos, lo cual equivale a un año de inactividad académica (Giovagnoli, 2002).

Dada la problemática se pretende analizar cuáles fueron principalmente las causas por las que los alumnos de la licenciatura en derecho generación 2016-2020 desertaron de la carrera.

El propósito de realizar esta investigación es conocer las razones, causas y motivos por los cuales los alumnos de la licenciatura en derecho abandonaron sus estudios, ya que existe la necesidad de conocerlas y analizarlas para poder buscar posibles soluciones y evitar este conflicto de gran importancia.

Los jóvenes, docentes y administrativos de cada una de las universidades del estado y del país se han percatado de que la deserción escolar es un problema social y educativo que puede afectar a cualquiera, debido a que los factores que lo provocan son muy comunes y se pueden presentar en cualquier ser humano, ya que todos pueden enfrentar problemas familiares, económicos y de salud diariamente provocando la desconcentración en los estudios y teniendo como resultado lo que es conocido como deserción escolar.

La deserción escolar es un conflicto que atañe e involucra a todos los individuos, es por ello que se decidió llevar a cabo esta investigación con la finalidad de estudiar las causas que orillan abandonar los estudios, específicamente las razones por las cuales los alumnos de la licenciatura en derecho abandonan su estancia en la universidad. Todo con el fin de poder evitar este problema de alguna manera y que por lo menos esta contrariedad ya no perturbe a la mayoría de los educandos.

Los propósitos de este estudio son analizar que motiva a los alumnos a abandonar la licenciatura en derecho. Describir que problemas son los más comunes que tienen que enfrentar los alumnos de la licenciatura en derecho para no desertar. Examinar cómo evitar la deserción escolar en los alumnos de derecho. Identificar en qué sexo sería más común que se presente la deserción.

\section{Materiales y Métodos}

La presente investigación conto con un estudio cualitativo, así como técnico-documental.

"La investigación cualitativa estudia la realidad en su contexto natural y cómo sucede, sacando e interpretando fenómenos de acuerdo con las personas implicadas." (Blasco \& Pérez, 2007) 
"La investigación documental es una disciplina instrumental, como cualquier actividad de tipo metodológica." (Tancara, 1993)

La población a investigar fueron los alumnos desertores de la generación 2016-2020 de la licenciatura en derecho. La muestra de población desertora se determinó tomando en cuenta la base de datos de control escolar en los diversos registros de inscripción y sistema de baja, el cual arrojo la cantidad de 18 desertores, de los cuales 7 son hombres y 11 mujeres y solo se pudo contactar a 14, con los cuales se realizó el trabajo de campo, tal y como se muestra en la Figura 1.

Al alumnado desertor, se le aplico un cuestionario de elaboración propia, dividido en 4 secciones, en los cuales se analizaron los factores económicos, vocacional, salud y familia. Dicho cuestionario se aplicó en el mes de abril del año 2020. Estudiando de esta forma el contenido de los cuestionarios para reconocer los motivos de la deserción escolar en la licenciatura en derecho con ayuda del programa operativo excel 2016.

\begin{tabular}{|l|l|l|l|l|l|}
\hline \multicolumn{5}{|c|}{ CUADRO 1. ESTUDIANTES DE LA LICENCIATURA EN } \\
DERECHO GENERACION 2016-2020 \\
\hline GENERACIÓN & $\begin{array}{l}\text { MATRICULA } \\
\text { INICIAL }\end{array}$ & $\begin{array}{l}\text { MATRICULA } \\
\text { FINAL }\end{array}$ & BAJAS & $\begin{array}{l}\text { NO } \\
\text { SIGUIERON } \\
\text { EL PROCESO } \\
\text { PARA } \\
\text { DARSE DE } \\
\text { BAJA }\end{array}$ & $\begin{array}{l}\text { DESERCIÓN } \\
\text { TOTAL }\end{array}$ \\
\hline $\mathbf{2 0 1 6 - 2 0 2 0}$ & $\mathbf{4 9}$ & $\mathbf{2 2}$ & $\mathbf{1 8}$ & $\mathbf{9}$ & $\mathbf{2 7}$ \\
\hline
\end{tabular}

Deserción en la generación 2016-2020

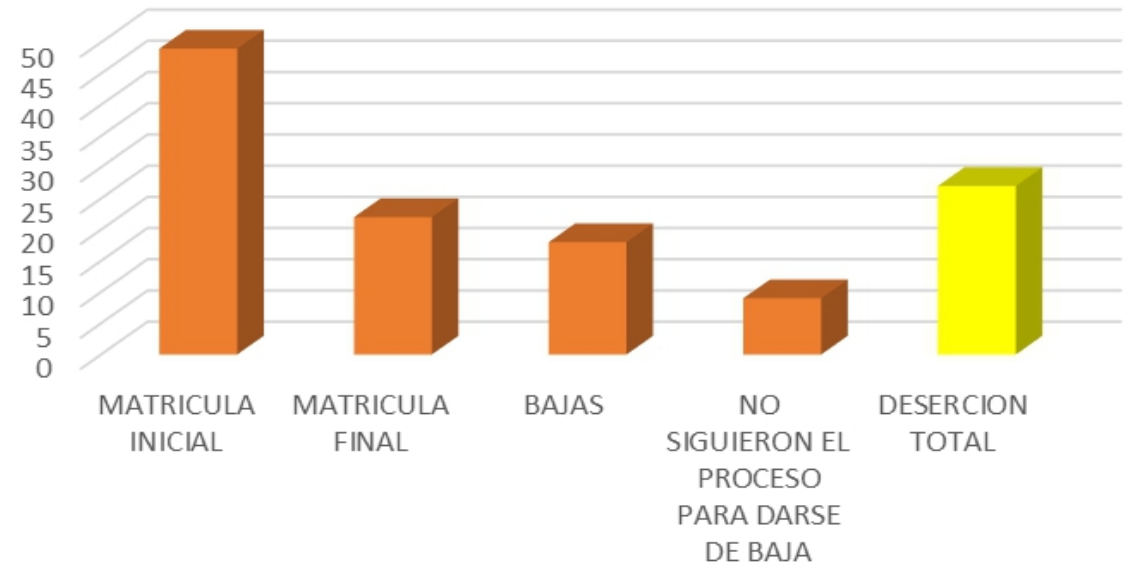

Figura 1. Total, de desertores en la generación 2016-2020 


\section{Resultados}

Conforme al estudio realizado a través de la consulta del registro de tutorías, en el registro de servicios escolares y en las listas de inscripción y sistema de baja, y a través del trabajo de campo realizado, se pudo comprobar que muchos de los alumnos que se dan de baja no se vuelven a inscribir e incluso que varios de estos se marchan sin darse de baja, y que estas situaciones se presentan debido al factor económico, vocacional, familiar y de salud.

\section{FACTOR ECONÓMICO}

Cuadro 2. Factores económicos que influyen en la deserción escolar de los estudiantes de derecho generación 2016-2020

\begin{tabular}{|l|l|}
\hline Factores & Porcentaje de cada ítem \\
\hline \begin{tabular}{|l|l|}
\hline Falta de dinero para las inscripciones & $25 \%$ \\
\hline Tenía que trabajar & $75 \%$ \\
\hline No tenía dinero para el transporte & $0 \%$ \\
\hline No tenía dinero para materiales escolares & $0 \%$ \\
\hline
\end{tabular}
\end{tabular}

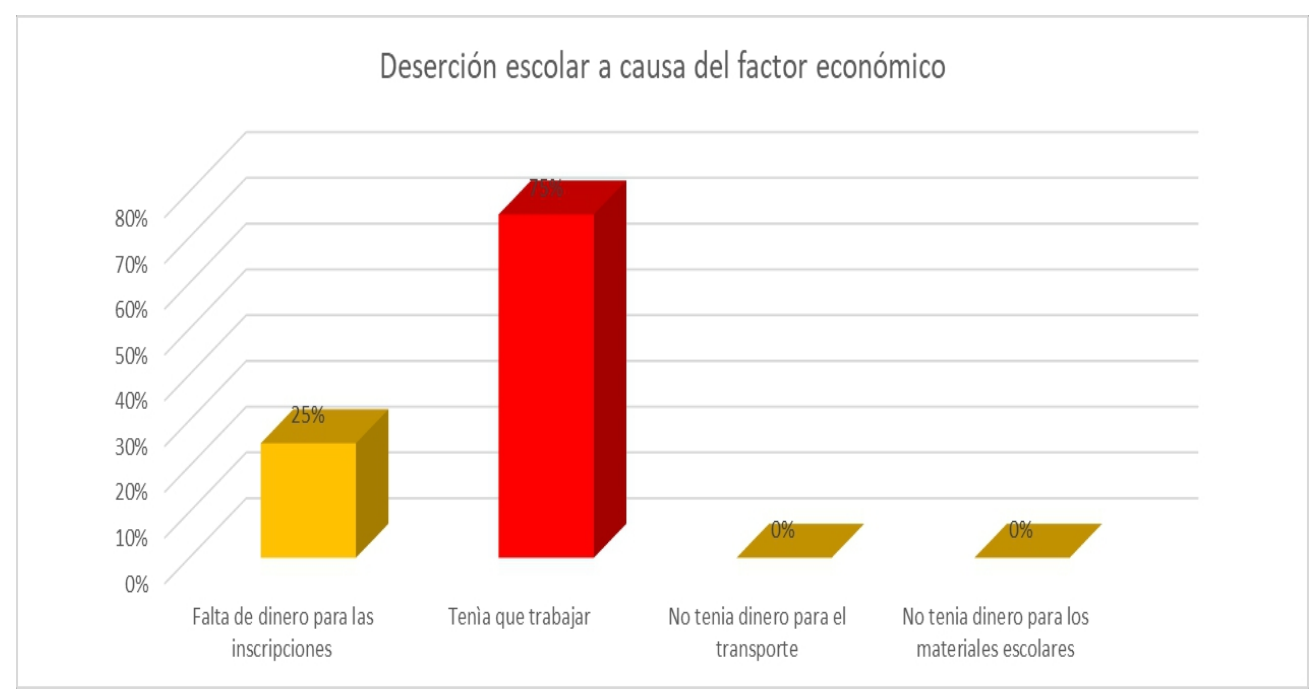

Figura 2. Deserción escolar a causa del factor económico

En el factor económico, las principales causas que influyeron se muestran en la Figura 2, las cuales son: tenía que trabajar con un $75 \%$ y falta de dinero para las inscripciones con un $25 \%$.

La economía influye debido a que como se sabe es el principal elemento de sobrevivencia, los gastos que se realizan en llevar a cabo una licenciatura no son mínimos de ahí su importancia como factor de deserción. 
Los alumnos deciden salirse de estudiar y comenzar a trabajar por la escasez de recursos, no se pueden realizar las dos cosas al mismo tiempo puesto que es muy pesado para ellos.

En este sentido es necesaria la presencia de más becas que pueden beneficiar a los estudiantes y aunque no sea suficiente, que con esto puedan sobrellevar la situación en la que se encuentran, ya que muchos de los jóvenes vienen de familias que se dedican al trabajo de campo y se complican más los problemas económicos.

\section{FACTOR FALTA DE ORIENTACIÓN VOCACIONAL}

Cuadro 3.- Factores por falta de orientación vocacional que influyen en la deserción escolar de los estudiantes de derecho generación 2016-2020

\begin{tabular}{|l|l|}
\hline Factores & Porcentaje de cada ítem \\
\hline & \\
\hline La licenciatura en derecho no fue su primera opción & $0 \%$ \\
\hline $\begin{array}{l}\text { La carrera que eligió no cumple con sus expectativas } \\
\text { Sus padres influyeron en la toma de decisión para la } \\
\text { elección de la carrera }\end{array}$ & $67 \%$ \\
\hline Eligió la carrera por su bajo costo & $0 \%$ \\
\hline
\end{tabular}

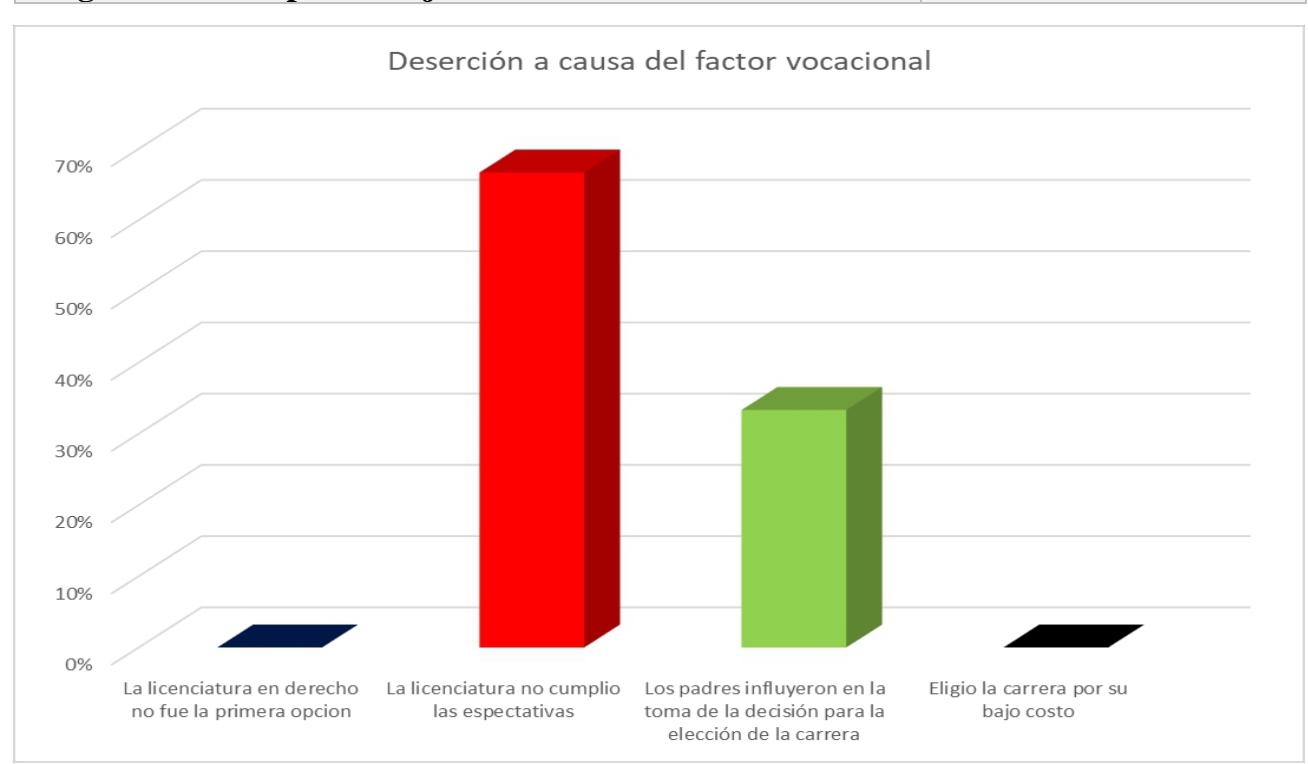

Figura 3. Deserción a causa del factor vocacional

En el factor vocacional, el principal motivo del abandono de los estudios fue que la licenciatura no cumplió con las expectativas de quien la eligió, con un $67 \%$ y como segundo factor esta que los padres influyeron en la toma de esta decisión con un $33 \%$. 
La falta de orientación vocacional es también un factor que interviene de gran forma, muchas veces se estudia algo no porque sea una primera opción o porque sea la correcta, más bien porque es la única, a veces los jóvenes se ven forzados a estudiar una carrera profesional porque es la más cercana a su domicilio, porque no es muy costosa, porque los padres deciden por los hijos o intervienen en la decisión de estos, o simplemente porque se toma una mala decisión debido a la falta de información.

Muchos alumnos se dan cuenta de que la carrera que eligieron no es de su gusto, pero esto sucede a causa de que no se orientan antes de hacer la elección, además se presenta debido a que los padres intervienen en la decisión que toman estos.

En este caso, deberían de contar con la ayuda de profesores y padres de familia cuando cursan la preparatoria o bachillerato para que tomen la decisión correcta, con tiempo y analicen o estudien de que trata cada carrera universitaria.

\section{FACTOR SALUD}

Cuadro 4. Factores de salud que influyen en la deserción escolar de los estudiantes de derecho generación 2016-2020

\begin{tabular}{|l|r|}
\hline Factores & Porcentaje de cada ítem \\
\hline
\end{tabular}

\begin{tabular}{|l|l|}
\hline Enfermedad propia & $67 \%$ \\
\hline Enfermedad de algún familiar & $0 \%$ \\
\hline Embarazo propio o de mi novia & $33 \%$ \\
\hline Muerte de mi papa o mama & $0 \%$ \\
\hline
\end{tabular}

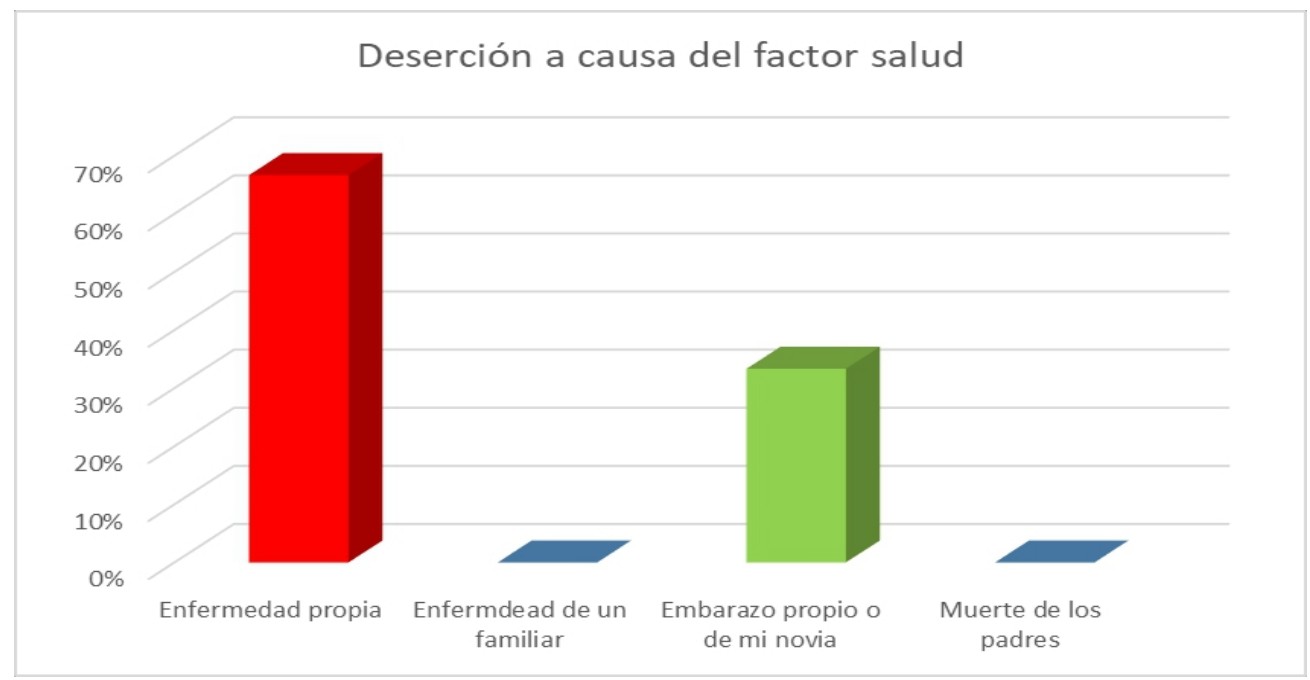

Figura 4. Deserción a causa del factor salud 
En el factor salud, las principales razones por las cuales los alumnos decidieron abandonar sus estudios fue la enfermedad propia con un $67 \%$ y con un $33 \%$ el embarazo propio o en la pareja a como se muestra en la Figura 4.

Las enfermedades son algo de lo que nunca podremos estar exentos, sin embargo, tratando los embarazos como parte de la salud, en este caso si se puede intervenir para que se reduzcan y no afecten a los estudiantes y así no sea motivo de deserción. Aunque siempre se hacen, se pueden hacer con mayor frecuencia las campañas sobre la utilización de métodos anticonceptivos para que de esta forma se evite éste factor, así como también brindando información y recordando que hay estudios psicológicos en la universidad con los que se puede contar para cualquier duda o inquietud.

\section{FACTOR FAMILIAR}

Cuadro 5. Factores familiares que influyen en la deserción escolar de los estudiantes de derecho generación 2016-2020

\begin{tabular}{|l|l|}
\hline Factores & Porcen \\
\hline Toda su familia tuvo que migrar & $0 \%$ \\
\hline $\begin{array}{l}\text { La religión que profesa su familia no le permite } \\
\text { continuar sus estudios }\end{array}$ & $0 \%$ \\
\hline $\begin{array}{l}\text { La familia se desintegro } \\
\text { Sus padres no lo apoyaron para seguir estudiando }\end{array}$ & $25 \%$ \\
\hline
\end{tabular}

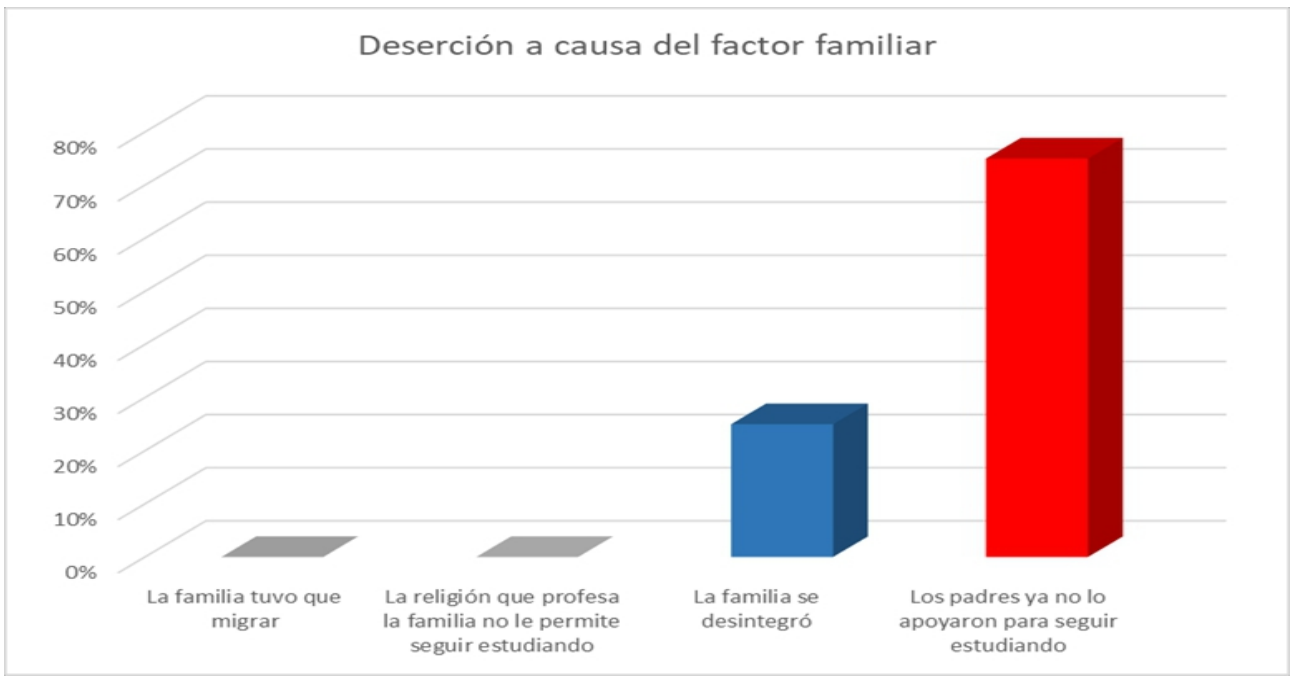

Figura 5. Deserción a causa del factor familiar

En el factor familiar, las principales causas de la deserción se muestra en la Figura 5 y estas fueron que los padres ya no los siguieron apoyando para seguir estudiando con el $75 \%$ y que la familia se desintegro con un $25 \%$. 
Como se puede deducir para estudiar una carrera universitaria se necesita de la motivación, si no se cuenta con esta no se concluyen los estudios, en la universidad la responsabilidad la tienen directamente los alumnos he ahí la importancia de estar motivado para no incumplir con las tareas y trabajos, no reprobar asignaturas ni dejar de asistir a las aulas.

Por eso importante contar con la ayuda de los padres, no solo económicamente hablando, sino también, con apoyo emocional. Que las familias se desintegren afecta psicológicamente a los jóvenes y al ver los problemas de los padres se desaniman, es por ello que esta situación provoca que los alumnos tomen la decisión de abandonar los estudios.

\section{Conclusion}

La deserción escolar es un problema educativo en el cual los alumnos abandonan sus estudios en el trayecto del ciclo escolar y es provocada por una serie de factores que se han identificado conforme a la investigación cualitativa y documental realizada, en base a datos obtenidos del trabajo de campo y de las oficinas de control escolar de las instalaciones de la Universidad Juárez Autónoma de Tabasco (UJAT), gracias a las cuales se indagó y permitió identificar que los alumnos de derecho que se dieron de baja de la universidad no regresaron, que los que si regresaron se atrasaron y ya no siguieron con la misma regularidad y que varios ni siquiera siguieron el proceso para darse de baja temporal.

Todo provocado por problemas multifactoriales, es decir, no se trata de una sola causa que intervenga en la decisión, son varios los factores que influyeron encontrándose entre éstos y en primer lugar la economía y la familia y en segundo lugar la salud y la falta de orientación vocacional, tal y como se planteó al inicio de esta investigación.

En el aspecto económico los jóvenes estudiantes de la carrera de derecho generación 2016-2020, tuvieron que abandonar sus estudios debido a que tenían que trabajar y a que no tenían dinero para las inscripciones.

En cuanto al motivo de deserción por razón de la familia, se debe a que esta se desintegra y a que los padres de los alumnos ya no quisieron seguir apoyándolos para que terminaran sus estudios.

En el factor salud, la mayoría de casos fueron debió a que las alumnas se embarazaron y a problemas de salud en los jóvenes. Y no se presentó ningún caso relacionado con la muerte o enfermedad de los padres.

Por ultimo en el factor vocacional, el abandono de la universidad, fue a causa de que, la carrera en derecho no cumplió con las expectativas de los alumnos y a que los padres de los jóvenes influyeron en la toma de decisión de los muchachos al elegir la licenciatura en derecho. 
Las formas de intervenir y mejorar esta situación están en manos de los alumnos, de los padres de éstos y por supuesto de la universidad, ya que ésta puede operar en sus propias instalaciones de acuerdo a sus posibilidades.

La máxima casa de estudios (UJAT), Universidad Juárez Autónoma de Tabasco, tiene como uno de sus principales ejes de desarrollo, la atención integral del estudiante, a través de una formación de calidad que propicie su permanencia en las aulas. Por ello cuenta con diversos programas de apoyo como el Programa Institucional de Tutorías y diferentes Becas, con los cuales los alumnos pueden apoyarse.

El Programa Institucional de Tutoría, adquiere relevancia, puesto que deriva del interés por apoyar a los estudiantes y favorecer su desarrollo integral, ya que con esta orientación se coadyuva en la permanencia del estudiante, abatiendo índices de rezago y deserción. El modelo concibe que la relación docente-alumno debe transformarse para aprovechar al máximo las potencialidades del estudiante, una formación integral en donde éste se convierta en el centro del proceso con la orientación de su tutor.

Los padres de familia deben enseñar a sus hijos los beneficios futuros de estudiar y atender las alertas cuando empiezan a dejar de asistir con regularidad a la universidad.

$\mathrm{Si}$ queremos que la carrera de licenciado en derecho forme profesionistas capaces de enfrentarse a los retos de nuestros tiempos, con buenas perspectivas de éxito, se debe apoyar a estos tanto económica y psicológicamente por parte de los padres, además de que estos no intervengan en la decisión de la elección de la carrera de sus hijos, así como también apoyarlos y apoyarlas en situaciones de embarazo y a prevenirlo. Por parte de los docentes orientar al alumno, a tomar las asesorías de su tutor, para que de esta forma tenga la oportunidad de tomar las decisiones para su formación profesional.

La deserción escolar es una contrariedad que vulnera la educación, los alumnos de cualquier carrera universitaria, en este caso la licenciatura en derecho, la sociedad y el desarrollo del país. Sin embargo, se puede contrarrestar si se atacan de lleno los factores que se ven involucrados y que han sido indagados y conocidos gracias a la investigación realizada.

\section{References:}

1. Arena, P. (2018, septiembre 05). Abandono e interrupción de estudios, la realidad de los universitarios en México. Arena Publica, p.3.

2. Arias, V. M. (2017, mayo 12). Deserción del 30\% por ciento anual registra la ujat. xeVT, $\mathrm{p}, 1$.

3. Aguilar, S. (2016). Informe de actividades damr. abril 19, 2020, de Universidad juárez autónoma de tabasco Sitio web: http://www.archivos.ujat.mx/2016/div_rios/informe-damr-2016.pdf 
4. Aguilar, S. (2017). Informe de actividades damr. Abril 19, 2020, de Universidad juárez autónoma de tabasco Sitio web: http://www.archivos.ujat.mx/2017/div_damr/2do-informe/2doinforme-damr.pdf

5. Aguilar, S. (2018). Informe de actividades damr. abril 20, 2020, de Universidad juárez autónoma de tabasco Sitio web: http://www.archivos.ujat.mx/2018/damr/3er-informe/3erinformedamr.pdf

6. Blasco, J. \& Pérez, J. (2007). Metodologías de investigación en ciencias de la actividad física y el deporte: ampliando horizontes. España: Editorial Club Universitario.

7. Castañeda, R. \& Rebolledo, C. (2014). Panorama de educación 2014. 20 julio, 2019, de Nota país, ocde Sitio web: http://www.oecd.org/education/Mexico-EAG2014-Country-Notespanish.pdf

8. Giovagnoli, P. (2002). Deserción estudiantil en la educación superior colombiana. Bogotá Colombia: Revolución educativa Colombia aprende.

9. Gracia, M. (2015, julio 22). Deserción universitaria en México. Milenio, p.2.

10. Liz, C. (marzo 25, 2011). Deserción estudiantil; Problema internacional, nacional, estatal y regional. 15 julio, 2019, de Deserción universitaria Sitio web:http://estresendesercionuniversitaria.blogspot.com/2011/03/antec edentes.html

11. Martínez, N. (2006, julio 31). Deserción universitaria causa frustración y pobreza: Unesco. El universal, p.2.

12. Narváez, G. (2020, enero 27). Deserción escolar multifactorial del $50 \%$ en la ujat: Guillermo Narváez. Corat, p.1

13. Nava, X. (2018, enero 10). Deserción, uno de los problemas vigentes en el sistema educativo de México: Nava. Pulso, p.1.

14. Páramo, G.J. \& Correa, C.A. (1999). Deserción estudiantil universitaria. Conceptualización. 15 julio, 2019, de universidad EAFIT Sitio web: file:///C:/Users/Isabel\%20Grrez/Downloads/1075Texto\%20del\%20art\%C3\%ADculo-3396-1-10-20120703.pdf

15. Rabelo, F. \& Aguilar, S. (junio, 2018). Reestructuración del plan de estudios de: licenciatura en derecho. Abril 19, 2020, de Universidad juárez autónoma de tabasco Sitio web: http://www.archivos.ujat.mx/2019/div-dacsyh/lic-derecho/Plan-deEstudio-Lic-en-Derecho.pdf

16. Ruiz, L. (2013, septiembre 23). Aumenta deserción escolar en la ujat. Rumbo Nuevo, p.1. 
17. Serrano, F. (2013, noviembre 28). Deserción universitaria en México: un problema que preocupa y que es un fracaso de todos. Universia, p. 1.

18. Tancara, C. (1993, diciembre). La investigación Documental. Instituto de Investigaciones Sociológicas SciELO, 17, p.2.

19. Vargas, I. (2013, enero 01). La deserción educativa rezaga a México. Expansión, p.2.

20. Ygartua, E. (2018, enero 19). Jóvenes abandonan sus estudios por problemas económicos, emocionales y adicciones. Diario Presente, p.4. 